Self-treatment with helminths

\title{
Socio-medical studies of individuals self-treating with helminths provide insight into clinical trial design for assessing helminth therapy.
}

Aarushi Venkatakrishnan ${ }^{1}$, Joshua T. Sarafian ${ }^{1}$, Kateřina Jirků-Pomajbíková ${ }^{2,3}$, William Parker ${ }^{1,4}$

${ }^{1}$ Department of Surgery, Duke University School of Medicine, Durham, NC, USA

${ }^{2}$ Institute of Parasitology, Biology Centre, Czech Academy of Sciences, 37005 České Budějovice, Czech

Republic

${ }^{3}$ Faculty of Science, University of South Bohemia, 37005 České Budějovice, Czech Republic

${ }^{4}$ Duke Global Health Institute, Duke University and Duke University Medical Center,

Durham, NC 27710, USA

Correspondence (present address): William Parker, Ph.D.

WPLab Inc.

1023 Wells St

Phone 919-949-5718

E-mail: William.Parker@,WilliamParkerLab.org 
Self-treatment with helminths

\begin{abstract}
The virtually complete loss of intestinal worms, known as helminths, from Western society has resulted in elimination of a range of helminth-induced morbidities. Unfortunately, that loss has also led to inflammation-associated deficiencies in immune function, ultimately contributing to widespread pandemics of allergies, autoimmunity, and neuropsychiatric disorders. Several socio-medical studies have examined the effects of intentional reworming, or self-treatment with helminths, on a variety of inflammation-related disorders. In this study, the latest results from ongoing socio-medical studies are described. The results point toward two important factors that appear to be overlooked in some if not most clinical trials. Specifically, (a) the method of preparation of the helminth can have a profound effect on its therapeutic efficacy, and (b) variation between individuals in the effective therapeutic dosage apparently covers a 10-fold range, regardless of the helminth used. These results highlight current limits in our understanding of the biology of both hosts and helminths, and suggest that information from selftreatment may be critical for clinical evaluation of the benefits and limits of helminth therapy.
\end{abstract}

Keywords: helminth; self-treatment; clinical trials; inflammation; dose escalation

Graphical Abstract:

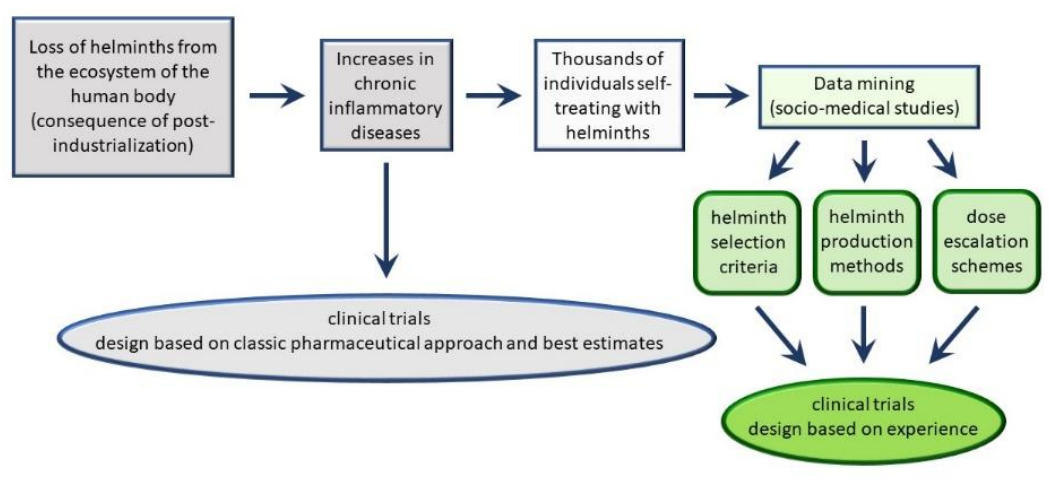


Self-treatment with helminths

\section{Introduction}

Intestinal worms, called helminths, have been almost completely eliminated in Western countries by widespread use of toilets, refrigerators, food storage technologies, and various food preservation techniques [1-3]. Although helminths in general cause a significant burden of disease, many helminths are benign [2] and, as a result, remain relatively unknown. Unfortunately, the loss of all helminths, both pathogenic and benign, from the ecosystem of the human body profoundly increases the propensity for chronic inflammation-associated disease in Western Society. The nature of immune system dysfunction caused by the loss of helminths is readily deduced based on the emergence of disease with the concomitant loss of helminths [4] and the effects re-introducing helminths into the population . For example, in the 1970s, seminal observations made by investigators in the UK demonstrated that exposure to helminths eliminated of allergic disease [5, 6]. Other studies conducted at the same time [7-10], confirmed by additional work decades later $[11,12]$, demonstrated that exposure to helminths prevents autoimmune diseases. More recent evidence has connected the loss of helminths with some inflammation-associated neuropsychiatric disorders, including a variety of anxiety disorders and major depressive disorder $[13,14]$. Further, numerous studies have elucidated a variety of immunological mechanisms underlying helminth therapy, including production of a wide range of helminth-derived immunoregulatory molecules, induction of regulatory networks, and activation of otherwise dormant immune components [15-20]. In light of this information, it is perhaps unsurprising that thousands of individuals today use helminths to treat their chronic inflammatory conditions [13, 21, 22]. 
Self-treatment with helminths

Systematic data gathering from people "self-treating" with helminths was first suggested by Flowers and Hopkins in 2013 [23] as an effective method for obtaining information regarding the effects of therapeutic helminths on patients with chronic immune related disease. Unfortunately, clinical trials approved by regulatory agencies probing the effectiveness of helminth therapy have been limited, in part by intellectual property rights [24] and regulatory issues [25]. Such issues are not easily overcome. For example, it may prove difficult to patent a naturally occurring organism for helminth therapy, and, as pointed out by a team of pharmaceutical industry specialists from Boehringer Ingelheim, one of the world's largest pharmaceutical companies, corporations cannot "shoulder the enormous costs involved" in clinical trials without patent protection for their investments [24]. Such investment without patent protection would potentially defraud investors and put the company at risk, both financially and legally. Further, current regulations are designed for testing of pharmaceutical compounds that are extremely well defined in terms of their physical/chemical properties. A given species of helminth, on the other hand, may be cultivated in dozens of ways and may have genetic variation. Characterization of the clinical impact of each individual variation would be a cost-prohibitive endeavor.

Unfortunately, as will be discussed in some detail in this manuscript, factors associated with formulation of therapeutic helminths may have profoundly impeded at least some clinical trials performed to date. Thus, at the present time, systematic data gathering from individuals selftreating may be the most practical and effective means of evaluating the effects of helminth therapy. Importantly, this view does not ignore the vital importance of clinical trials, which ultimately must be conducted before helminth therapy can be utilized in main-stream medicine.

Previous studies collecting information from individuals self-treating with helminths $[13,21$, 22] have encompassed four helminths, a wide range of disease, and more than 1000 individual 
Self-treatment with helminths

cases. Data collection methods include surveys, interviews with helminth providers, and interviews with physicians who have experience observing patients who are self-treating. Information concerning the use of four helminths has been collected: the porcine whipworm (TSO), the human whipworm (TTO), the human hookworm (NA), and the rat tapeworm (HDC). This assortment of helminths was "selected" for use by self-treaters more by happenstance than by design [26], but provides a range of alternatives for self-treaters in terms of both financial costs and benefit/risk ratios [26].

Prior studies evaluating the outcomes of individuals self-treating with helminths [13, 21, 22] have utilized a series of methods, including surveys for self-treating individuals, surveys for physicians who monitor self-treating individuals, and interviews with producers and suppliers of helminths. In some cases, survival bias and response bias have been either eliminated or quantified, and several lines of evidence, outlined in the Discussion, indicate that placebo and nocebo effects contribute minimally to reported outcomes. Conclusions are considered strongest when supported by multiple approaches, and especially in cases where results of self-treatment match published clinical observations. For example, prospective studies evaluating the effect of accidental helminth colonization on relapsing remitting multiple sclerosis $[11,27]$ have corroborated studies in individuals self-treating their multiple sclerosis with helminths [13, 21]. In addition, clinical observations [5] have corroborated positive results obtained by individuals self-treating their seasonal allergies with helminths [13, 21]. Other conclusions based on evaluation of individuals self-treating with helminths have been validated in studies using laboratory animal models. For example, studies in animal models [9, 10], have corroborated positive results obtained by individuals self-treating with helminths to alleviate autoimmune conditions such as lupus and some cases of arthritis [13, 21]. Studies in animal models [14] have 
Self-treatment with helminths

also corroborated positive results obtained by individuals self-treating with helminths to alleviate neuropsychiatric disorders $[13,22]$.

Although self-treatment with helminths is apparently beneficial to many individuals with some disorders, studies of individuals self-treating with helminths have revealed limitations to the therapy. For example, self-treatment with helminths may effectively treat relapsing remitting multiple sclerosis and alopecia areata $[13,21,22]$, but may not be as effective at treating more advanced stages of these conditions, progressive multiple sclerosis and alopecia totalis. Further, helminth therapy may effectively treat seasonal allergies, but may have little beneficial effect for individuals suffering from chronic exposure to household mold. Thus, studies of individuals selftreating with helminths reveals benefits as well as limitations to the therapy.

In this study, we provide a series of new findings that add to our previous socio-medical studies. Importantly, new information highlights the importance of two factors that may not be adequately considered in some recent clinical trials: (a) individualized dosage requirements and (b) helminth preparation and storage conditions. These findings suggest that the current "gold standard" trials [28], despite their double-blind, placebo-controlled design, are providing lackluster or even false negative results due to a lack of consideration of the biology of helminths and their interactions with their hosts. That being said, helminth therapy is not expected to resolve all chronic inflammatory conditions, an expectation supported by studies of individuals self-treating with helminths. Thus, as we begin to understand and implement effective design of clinical trials for helminth therapy, limitations to the therapy will doubtless emerge as benefits to the therapy are identified. 
Self-treatment with helminths

\section{Methods}

\subsection{General methods}

Studies were approved by the Duke Institutional Review Board. All research was performed in accordance with relevant guidelines/regulations, and informed consent was obtained as dictated in the approved protocol. At no time was any protected health information gathered, and no personally identifying information was collected, ensuring anonymity of the participants. This study, as with our previous studies $[13,21]$, utilized multiple approaches to probe the outcomes of self-treatment with helminths. As previously described [21], the multiple methodologies facilitated acquisition of more diverse information than would have been obtainable with a single method alone, and allowed triangulation between methods to strengthen conclusions regarding some aspects of the practice of selftreatment with helminths. In this study, three approaches were used to evaluate current practice and outcomes in self-treatment with helminths. First, individuals prominent in the self-treatment community were interviewed. These included individuals producing, selling, and/or distributing helminths ("providers") for self-treatment with helminth therapy, and individuals compiling and organizing data regarding outcomes of self-treatment in a public format. As a second approach, publicly available information regarding self-treatment with helminths was compiled and evaluated. Third, physicians (all board-certified MDs) who see patients that self-treat with helminths were interviewed.

\subsection{Assessment of dosing recommendations by evaluation of publicly available information}

To provide information regarding the range of hookworm doses used for self-treatment, publicly available, self-reported personal experiences with dose adjusting using the human hookworm were examined. This approach does not control for survivor bias, and is thus not useful as an absolute measure of effectiveness of helminth therapy. Further, the data obtained cannot be taken as evidence that helminth therapy actually "works", or has a good benefit to cost ratio when applied by knowledgeable practitioners. The view that helminth therapy works is based on other studies, as described in the Introduction. Finally, additional caveats to this approach need to be kept in mind, some of which are described in the Results 
Self-treatment with helminths

section. Nevertheless, the approach was useful in probing the range in effective dose of hookworm as reported by self-treaters. Helminthic Therapy Wiki ("Helminth Wiki”), a popular crowd-sourced site that consolidates information about helminth therapy, was used as an initial source of information. Most of the reports on Helminth Wiki came from a Yahoo group, "Helminthic Therapy Forum," and the "Helminthic Therapy Support" Facebook Group. While the Yahoo group was the longest active forum for helminth users, in December 2019 Yahoo removed all user content from its groups, leaving the information to be solely held on Helminthic Therapy Wiki. In the Facebook group, posts between January 2012 and December 2019 in were reviewed and users' personal experiences with dose adjustments were recorded. Two illnesses with the highest number of selected cases on the Wiki site were evaluated: Allergy and Crohn's colitis. Independent searches were conducted using the keywords "Allergy" or "Crohn's" and modifying the parameters to focus on a specific year in the date range. Cases were omitted if (a) the physical condition was not either allergies or Crohn's colitis, (b) the dose of helminths used was not specified, or (c) any helminth other than the hookworm was used, even if it was used in combination with the hookworm. Since this study was focused on the dosage used for effective therapy, cases that did not report a positive effect of helminth therapy were also excluded. In all remaining cases, the doses of helminth used were recorded and analyzed.

\section{Results}

\subsection{General}

The four helminths currently used for self-treatment are the same ones that have been used for about 7 years. The most recent addition for self-treaters was the rat tapeworm (HDC) in 2013. However, the popularity of various helminths continues to shift, with the human whipworm (TTO) becoming less popular due to a relatively high cost/benefit ratio compared to other helminths, and a lack of advantages over other helminths (Table 1). The observation that HDCs appear to be an effective therapeutic substitute for TTO was made by one of the most experienced helminth suppliers and has not been 
Self-treatment with helminths

previously reported. Given that HDCs have a lower risk profile than TTO, it is anticipated that HDCs will continue to increase in popularity, while TTO decreases in popularity. However, the choice between different helminths for most individuals continues to be a balance between higher-cost helminths (TSO and HDC) that do not effectively colonize humans, and lower cost helminths that effectively colonize humans (TTO and NA). Further, it is expected that individuals who have had success with TTO in the past will continue to use the organism for therapeutic purposes.

\subsection{Growth of self-treatment}

No therapeutic helminth has yet been approved for use by the FDA. Further, at least two suppliers have tried to obtain approval through regulatory agencies for helminths as a novel food. These efforts have thus far not met with success. Nevertheless, self-treatment with helminths has continued to grow as judged by the number of companies currently providing helminths. The total number of helminth suppliers more than doubled between 2014 and 2020 (Figure 1). A list of current suppliers can be found in Table 2. The table excludes commercial suppliers who informed the study team that they did not wish to be publicly identified. In addition, "helmTX" is not included. Although they describe their helminth as "FDA approval pending", they do not currently provide helminths for self-treaters. Suppliers include some established brick and mortar facilities (e.g., Tanawisa, Biome Restoration, Worm Therapy, The Llamas Clinic) as well as others who can only be found on the internet or via Email (e.g., Autoimmune Therapies, Au Naturel, YourSymbionts). As shown in Figure 1, the number of suppliers providing HDCs and NA has expanded considerably. TSO, on the other hand, continues to be produced only by Tanawisa.

\subsection{Publicly organized data: Helminth Therapy Wiki}

Information regarding helminth therapy from social media groups such as Facebook and Yahoo has been available to the public for a number of years [21]. Perhaps the biggest development in terms of social organization for self-treaters is the extensive development of a website designated exclusively for helminth therapy results. This website, "Helminthic Therapy Wiki”, is regularly curated, gleaning 
Self-treatment with helminths

information from the web as well as published information related to helminth therapy. The website compiles information on suppliers, financial costs of therapy, financial arrangements used by various suppliers (subscription, one-time payment, or contracts), treatment regimens, and results.

\subsection{One size does not fit all: the effective dose of helminth varies from individual to individual}

All helminths for which sufficient data from physicians and helminth suppliers have been obtained (HDCs, NA, and TSO) indicate that the effective dose of a given helminth is highly variable from individual to individual. The effective dose ranges for HDCs, for NA, and for TSO each vary by more than 10-fold, and are shown in Table 3.

A process of "dose-finding" for helminths has been developed and is recommended by most suppliers of helminths. In addition, six out of the seven physicians interviewed who provide information to patients self-treating also supported the dose-finding approach as being safer than attempting therapy with a "standard" dose defined as an effective dose for an average individual (Table 3). The dose finding approach entails starting with a low dose of helminth and then slowly working up to higher doses until either (a) symptom relief is obtained or (b) adverse side effects are sufficient to warrant backing off on the dosage or cessation of the protocol. This dose-finding protocol has been described previously for HDCs [22]. Dose finding is also readily achieved for TSO given its stability during storage in a liquid suspension and relative frequency of administration on a weekly basis. However, it was noted by two suppliers that dose finding for NA can be time prohibitive. Because maturation of hookworm in the human body is a time-consuming process requiring 6 to 8 weeks to achieve peak effects on immune function [29], and because approximately three months are often recommended between doses of NA for self-treatment, a dose finding scheme involving gradual escalation of NA could take years. In cases where the disease state is debilitating or progressive, this time frame may be unacceptable. Indeed, the single physician who did not support dose finding by gradual escalation of exposure worked exclusively with 
Self-treatment with helminths

patients self-treating with NA. That said, another physician interviewed was adamant that failure to start at low exposure levels of NA was irresponsible and fraught with problems.

Assessment of publicly available information regarding the treatment of allergies and Chron's colitis using NA (see Methods) was used to glean further information regarding the dose range for NA. As shown in Figure 2, the number the organisms reported to be effective varied over a 10-fold range, both for the treatment of allergies and for the treatment of Crohn's. This observation held true for both the starting dose and the total number of helminths taken over time. Perhaps unexpectedly, the average effective dose did not vary significantly as a function of disease (Figure 2).

A significant but weak association was observed between the starting dose and the cumulative dose per year (Figure 3), suggesting that some hesitancy on the part of some individuals rather than objective dose-finding schemes may underlie their relatively lower doses. However, because only successful cases were considered, it also seems likely that some unknown number of individuals starting at higher doses were excluded due to adverse events. With that in mind, those individuals starting at the higher doses may be self-selected for individuals who tolerated higher doses, thus providing a correlation between starting dose and cumulative dose. Nevertheless, correlations between initial doses and cumulative dose per year were not strong, indicating that many individuals adjust their dose either up or down during the course of their treatment with NA (Figures 2 and 3).

\subsection{Miscellaneous new observations on the practice of helminth therapy}

As mentioned above, one important new observation made by a supplier with extensive experience with multiple organisms was that HDCs are apparently as effective as TTO for treatment of ulcerative colitis, the primary indication for TTO. If this observation bears out, it is encouraging news, since the HDC has a better adverse side effect profile than does TTO. The same supplier also observed that extensive washing of NA for the purpose of removing bacteria prior to administration can profoundly 
Self-treatment with helminths

diminish its effectiveness. Since cleaning the organisms is now standard for all FDA-approved trials, this observation might suggest that doses of NA used by self-treaters (e.g., Table 3, Figure 2 and 3) may be insufficient in clinical trials.

Cases of individuals being colonized with therapeutic HDCs continue to surface, although they are not common, and they occur primarily in children or in immunosuppressed adults. One case of a child being colonized with TSO has been reported through our socio-medical study, and it is believed that this case did not involve cross-contamination with TTO. (TTO and TSO can easily be mistaken for each other without appropriate assessment using genetic tools.) Although some cases of colonization of adults with TSO have been reported in the literature [30-32], R. J. Beer in 1976 described the eggs produced by TSO in humans as being unhealthy or "degenerate", and described the colonization as benign (without overt pathology). Further, different strains of TSO exist [33], and it is unknown whether specific characteristics of the strain used for therapy might affect colonization tendencies in humans. Nevertheless, it seems likely that none of the current helminths in use are completely non-colonizing in humans. Persistence in some cases may be expected, even if it is rare. Since these organisms can in rare cases persist long enough to produce eggs, they should be described as "poorly colonizing" or "weakly colonizing" rather than "non-colonizing". Nevertheless, self-treatment using TSO or using HDCs has never, to our knowledge, resulted in colonization in an adult without active immunosuppression. Furthermore, given that colonization with TSO and HDCs in children is limited to a hand-full of cases despite hundreds of self-treating children, and given that children intentionally exposed to helminths typically have chronic conditions associated with immune dysfunction, it might be hypothesized that the rate of colonization of children with uncompromised immune systems by these organisms is vanishingly small.

NA has frequently been administered on the forearm. However, treatment with NA by most selftreaters now utilizes application to the feet rather than the forearm. The view that this approach causes substantially less irritation at the site of entry of the organisms was corroborated by two physicians and one supplier. NA has a strong affinity for human skin [34], and is transferred from host-to-host via 
Self-treatment with helminths

exposure of the skin to soil containing the organisms [35]. With this in mind, it seems likely that the natural point of entry into human skin would likely be areas on the body that are calloused due to regular contact with soil. The same supplier who noted that NA washed free of bacteria is less effective than unwashed NA (see above) also noted that NA washed free of bacteria caused less irritation at the site of entry. While this observation could be attributed to damage or attenuation of the NA during the washing procedure, it might also suggest that the bacteria carried with the NA are capable of causing irritation as the helminth moves through the skin. If that is correct, then it seems likely that, when NA enters the skin through a calloused area of the body, some of the microbial components are deposited in the callous, resulting in decreased irritation when the NA reaches a vascularized area.

Self-exposure to NA was reported by one helminth supplier to alleviate most $(\sim 70 \%)$ of the symptoms of narcolepsy without cataplexy, consistent with the previously held view [36] that helminth therapy can treat a wide range of neurological disorders associated with chronic inflammation, including anxiety disorders and major depressive disorder [13].

Mast cell disorders and fibromyalgia have previously been reported to be contra-indications for helminth therapy due to adverse reactions to the symbionts in patients with these conditions [21]. This conclusion has been corroborated by an additional supplier. In addition, a supplier with extensive experience in the application of helminth therapy and with ties to a clinic treating patients with helminths reports that fistulating Crohn's disease is also a contra-indication for helminth therapy.

\subsection{A persistent question: Does preparation and storage of TSO affect its efficacy?}

TSO is the organism that has been most often used by individuals self-treating with helminths [21]. Based on socio-medical studies [13,21], we have concluded that, consistent with earlier successful trials $[37,38]$, TSO is indeed effective. Roughly a third of patients with a wide range of chronic inflammatory conditions experience "profound" or "dramatic" improvements of chronic conditions, with less than $1 \%$ of individuals experiences adverse reactions worse than temporary gastrointestinal upset. However, the therapeutic effect of the organism has not born out well in clinical trials [39, 40]. An 
Self-treatment with helminths

explanation for these conflicting observations is adamantly supported by the supplier of TSO. Prior to the report of the first failure of a clinical trial with TSO, our socio-medical study received reports from the supplier that clinical trials utilized organisms that were poorly prepared, thereby decreasing their efficacy [21]. The central question regarding preparation of the organism deals with the acidity of the solution in which the organisms are suspended: $\mathrm{pH}$ of storage, how fast the $\mathrm{pH}$ is changed prior to administration to patients, and how long it sits at a particular $\mathrm{pH}$ prior to administration.

As mentioned above and discussed previously [21], controversy regarding the preparation of TSO centers around the $\mathrm{pH}$ of the preparation. During the initial isolation of TSO in preparation for clinical use, the $\mathrm{pH}$ is lowered dramatically ( $\mathrm{pH}$ approximately 1.0) to inactivate pathogens that may be present in the porcine feces from which TSO is harvested. Then the $\mathrm{pH}$ is raised at least to $\mathrm{pH} 2.5$ prior to storage. This process differs substantially from the $\mathrm{pH}$ encountered by the organism during its natural life cycle, in which a low $\mathrm{pH}$ is only temporarily encountered in the distal porcine stomach immediately following ingestion (Figure 4). As shown in Figure 4, the $\mathrm{pH}$ of therapeutic preparations of TSO can vary dramatically and depends on the preparation. Further, the method of raising the $\mathrm{pH}$ following initial preparation can vary, and the length of time in storage after raising the $\mathrm{pH}$ can vary. The manufacturer of TSO for self-treatment contends that only by raising moderately (i.e., up to $\mathrm{pH} 2.5$ ) and slowly can effective TSO be maintained. Based on their customer feedback, they contend that raising the $\mathrm{pH}$ to as high as 5.5 prior to storage can render the TSO ineffective over time. Despite the fact that TSO has been used in clinical trials, the effect of $\mathrm{pH}$ on the viability of the organism and its ability to hatch has not been investigated. Some work has been done with the mouse whipworm [41], but results from studies using the mouse whipworm may not apply to the porcine whipworm [42].

Given that helminth suppliers often receive regular feedback on their preparations from their customers, their measure of efficacy can be both extremely rapid and clinically relevant. Individuals running clinical trials, on the other hand, must rely on surrogate markers, such as the hatching of the organisms in an animal model. Unfortunately, at least for the HDC, measures in laboratory animals are 
Self-treatment with helminths

ineffective. Several factors, including advanced age of the organisms and feeding extremely rich diets to their hosts, apparently harm the therapeutic benefit of the organisms [22] without impeding the ability of the organisms to mature in their native hosts. It is indeed difficult to imagine how such sensitivity to the conditions of preparation could be elucidated in clinical trials. The argument has been made that, because the organisms produce some adverse side effects in clinical trials, they must be viable. While this argument certainly has merit, the nature of the side effects is of interest. As an example, Figure 5 shows intestinal distress reported in one trial [43]. In that trial, in which TSO proved to be ineffective at the dose used, a significant amount of GI distress in most patients following the second dose of the organism was observed, although the GI distress did not persist despite continued treatment. It is unclear whether this temporary increase in side effects demonstrates that the organisms used for the duration of the trial were active, and it is also unclear whether this side effect correlates with the potential for clinical effectiveness.

\section{Discussion}

At the present time, several clinical trials, reviewed recently [28], have been published using TSO [39], while others have been published using NA [29, 44]. Despite (a) numerous positive results in animal models, (b) positive results reported for individuals self-treating with helminths, and, in some cases, (c) positive reports from clinical examination following accidental exposure to helminths $[5,11,27]$, results in clinical trials have been disappointing [40].

Reports from individuals self-treating with helminths, from physicians treating those patients, and from individuals producing therapeutic helminths provides a potential explanation for the lack of positive results in clinical trials: investigators have not taken into account the greater than 10-fold dose range, depending on the individual, that is apparently required to achieve a satisfactory effect. This issue applies to all potentially therapeutic helminths studied to date (Table 3), and presents considerable challenges to clinical trials that would incur considerable 
Self-treatment with helminths

time and expense with the implementation of any lengthy dose-finding scheme. For example, one trial with NA used one exposure to 10 organisms over a 16-week period [29], whereas another study used two exposures to, one of 10 organisms and another of 5 organisms, over a 20week period [45]. This level of exposure falls considerably below levels used by many individuals self-treating with helminths (Figure 2, Table 3), suggesting that it may not be adequate for many individuals. At the same time, higher levels of exposure probably should not be attempted study wide, as such levels would likely cause adverse reactions in many individuals.

Unfortunately and perhaps unavoidably, investigators have apparently been largely unaware that the effectiveness of therapeutic helminths can depend on the production method and storage conditions. This issue is a factor for HDCs and probably for TSO and NA as well. Based on this assessment, we conclude that the conduct of clinical trials needs to be altered significantly, taking into account the biology of helminths as well as the variable immunology of their hosts. If the current trends continue, it seems possible that the therapeutic potential of helminths will be overlooked in favor of pharmacological therapies that are more expensive and potentially less effective. Of considerable concern in this regard is the view that exposure to benign helminths is probably a reasonable preventative measure, whereas treatment with helminth derived products is expected to be utilized only after disease has occurred.

A variety of factors indicate that results reported to us through socio-medical studies are representative of the actual effects of helminth therapy, not artifacts due to placebo effects, normalization to the mean, survivor bias, conflicts with commercial interests, or other issues unrelated to the actual benefits and risks of the therapy. These factors are as follows: 
Self-treatment with helminths

- Survivor bias has been eliminated using some data collection methods (e.g., interviews with physicians and a survey collection method supported by a helminth supplier).

- Reviews, both positive and negative, made by individuals (typically experienced physicians or suppliers) with multiple observations are consistent and corroborating (independent of the commercial interest of the source).

- Reported beneficial effects of therapy are long lasting if the therapy is maintained, inconsistent with the placebo effect.

- Therapy is reported to effectively treat chronic conditions present for decades and despite multiple failures with pharmaceutical approaches, inconsistent with normalization to the mean.

- Therapy has been used to treat degenerative conditions that are irreversible, such as multiple sclerosis, inconsistent with normalization to the mean.

- Beneficial effects of therapy often surprise the users, inconsistent with the placebo effect.

- Therapy has been reported to be effective in small children, who are not prone to influence by expectations or preconditioning.

- Self-treaters indicate that they can tell when their beneficial helminths lose effect and acquisition of more organisms is required. The timing is helminth specific, indicating that the reports are based on a phenomenon related to helminth biology, not human psychology.

- The effects reported by self-treaters are supported by numerous studies in animal models.

The adverse side effects associated with uncontrolled colonization with helminths and, perhaps to a lesser extent, clinical trials with helminth therapy have led some research groups to 
Self-treatment with helminths

pursue helminth derived products (HDPs) rather than helminths themselves as a treatment for inflammatory diseases [46, 47]. In January 2020, Capron et al. published the first clinical trial to evaluate the safety of the recombinant helminth protein, P28GST from Schistosoma haematobium, in patients with mild or inactive Crohn's disease [48]. P28GST was first developed as a vaccine against schistosomiasis, however, researchers have found that the protein has a positive effect in humans and other animal models against inflammation [48-51]. Through their pilot study, they found that the patients experienced a positive response and they assert that using HDPs are a more promising approach as they can replicate the benefits of helminthic therapy without the detriments. The view is that, because the immunomodulatory effectiveness of helminths can be attributed to their surface or excretory/secretory products, therapies can be designed using only these key products [52]. Thus, some researchers, while recognizing the potential benefits of helminthic therapy, have turned away from the use of live helminths and instead pursued HDPs [52-54]. At the present time, however, for theoretical reasons, we do not agree that this approach is warranted: Specifically, we predict that the complexity of the immunoregulation derived from the helminth/host relationship will be impossible to replicate in the foreseeable future using individual components derived from helminths. Here we describe evidence that the efficacy of helminth therapy can be improved and the adverse effects of the therapy can be reduced using personalized dose adjustments over time. Further, we continue to argue that controlled, moderate exposure to domesticated, benign helminths is a practical and cost-effective solution to undermining increases in a variety of hyper-immune related diseases in Western culture [1].

\section{Conclusions}


Self-treatment with helminths

Helminth therapy addresses a fundamental cause of chronic inflammatory disease in Western society. In particular, helminth therapy reverses the loss of helminths in Western society that has left the immune system overreactive and prone to allergic, autoimmune, and neuropsychiatric disorders $[17,55]$. At the present time, we would argue that the primary "lab" for helminth therapy seems to be within the community of self-treaters. This "biohacking" phenomena of science outside of the established main stream is not unique to helminth therapy. However, given that helminth therapy addresses a fundamental cause of disease in Western society, this particular biohacking endeavor may in fact be critical for public health. Unfortunately, for a variety of economic, regulatory, and practical issues surrounding the conduct of clinical trials, main stream trials have thus far been unable to accommodate the nuances of helminth therapy. Foremost among the issues that clinical trials must address before they can effectively test the potential for helminth therapy are (a) details in formulation of the helminth product that affect efficacy, and (b) the very wide range of doses typically needed within a cohort of individuals.

\section{Acknowledgements}

The authors would like to thank the physicians, suppliers, and others knowledgeable regarding the self-treatment with helminths for altruistically providing the information compiled in this manuscript. The authors also wish to thank John and Susan Poulton for their encouragement and support of this work. This work was supported by the project LTAUSA19008 funded by the programme INTER-EXCELLENCE of the Ministry of Education, Youth and Sports of the Czech Republic. 
Self-treatment with helminths

Figures and Figure Captions.

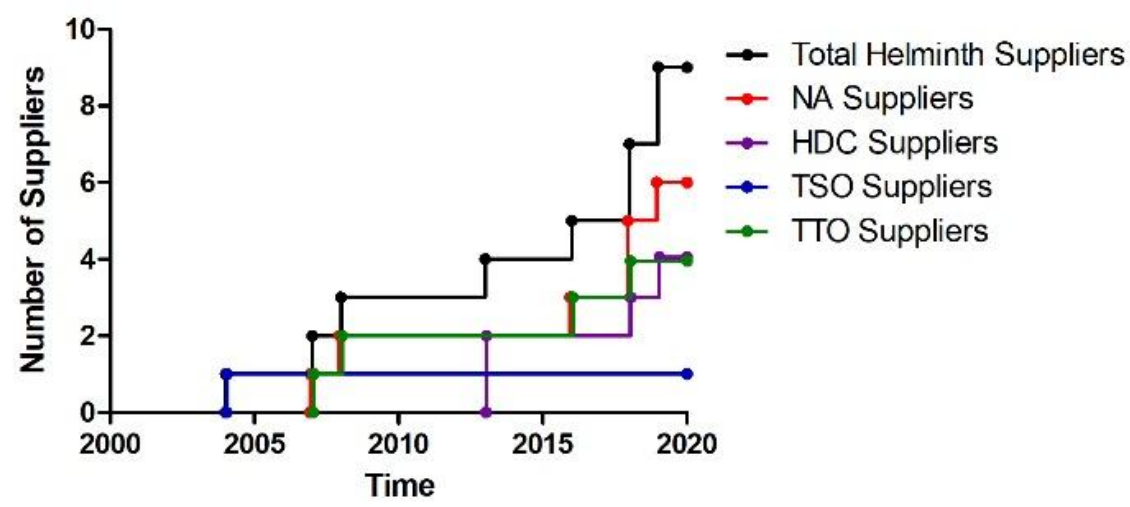

Figure 1. Emergence of companies selling various helminths. Only companies currently supplying helminths are shown on the graph; companies no longer in business are excluded. In addition, companies which do not wish to be recognized publicly are not shown on the graph. 
Self-treatment with helminths

A

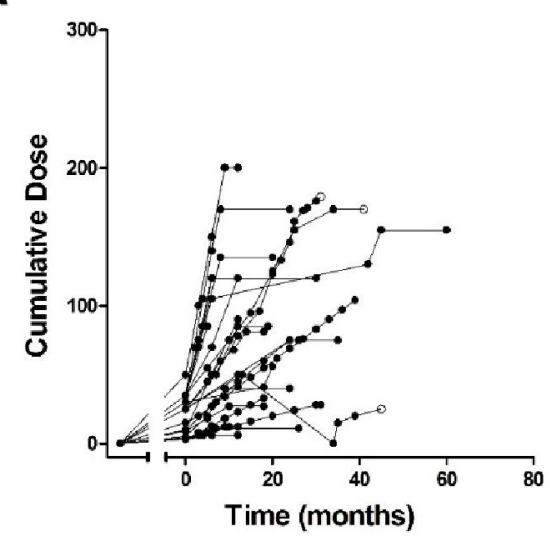

C

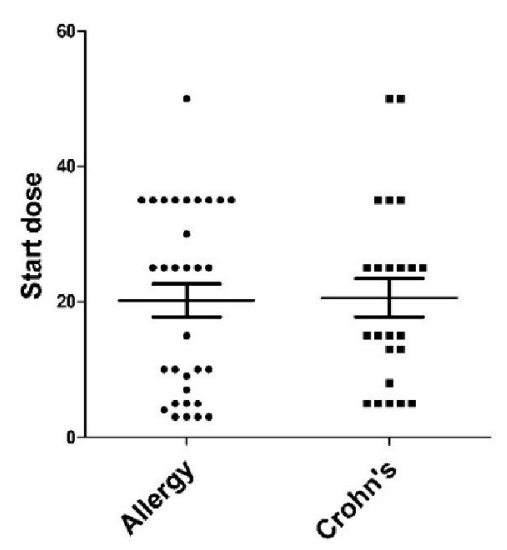

B

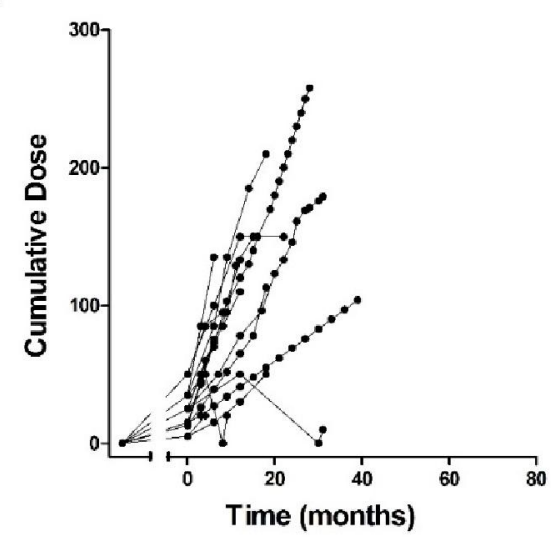

D

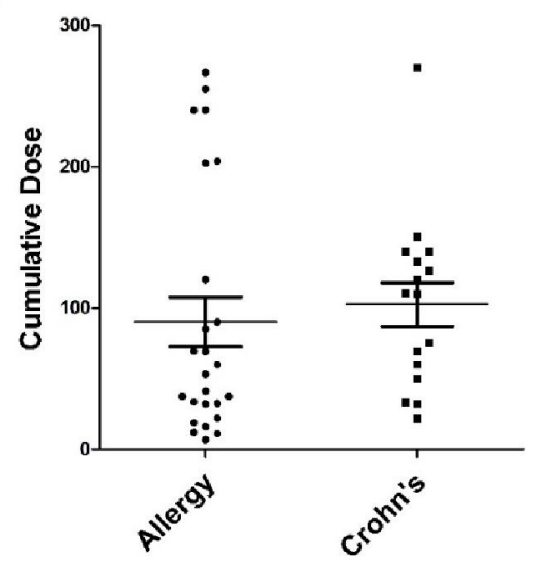

Figure 2: Variation in dosage of the human hookworm (NA) from self-reported, successful treatment of allergies and Crohn's colitis. The self-reported dosing strategy taken by individuals with allergies $(\mathrm{n}=25)$ and Crohn's $(\mathrm{n}=16)$ is shown in panels A and B, respectively. Open circles represent instances where an individual determined that their dose was too high and started to worsen their condition. Panel $\mathbf{C}$ shows a comparison between the starting dose of NA taken between self-treaters with allergies $(n=32$, mean $=20.22$, std deviation $=13.88$, std error $=2.454)$ and those with Crohn's $(\mathrm{n}=23$, mean $=20.61$, std deviation $=13.62$, std error $=2.841) \mathrm{p}=0.8696$. Panel $\mathbf{D}$ compares the cumulative dose, normalized per year, for self-treaters with allergies $(n=25$, mean $=$ 90.25, std deviation $=86.86$, std error $=17.57)$ and those with Crohn's $(n=16$, mean $=102.6$, std deviation $=62.04$, std error $=15.51) \mathrm{p}=0.1857$. 
Self-treatment with helminths
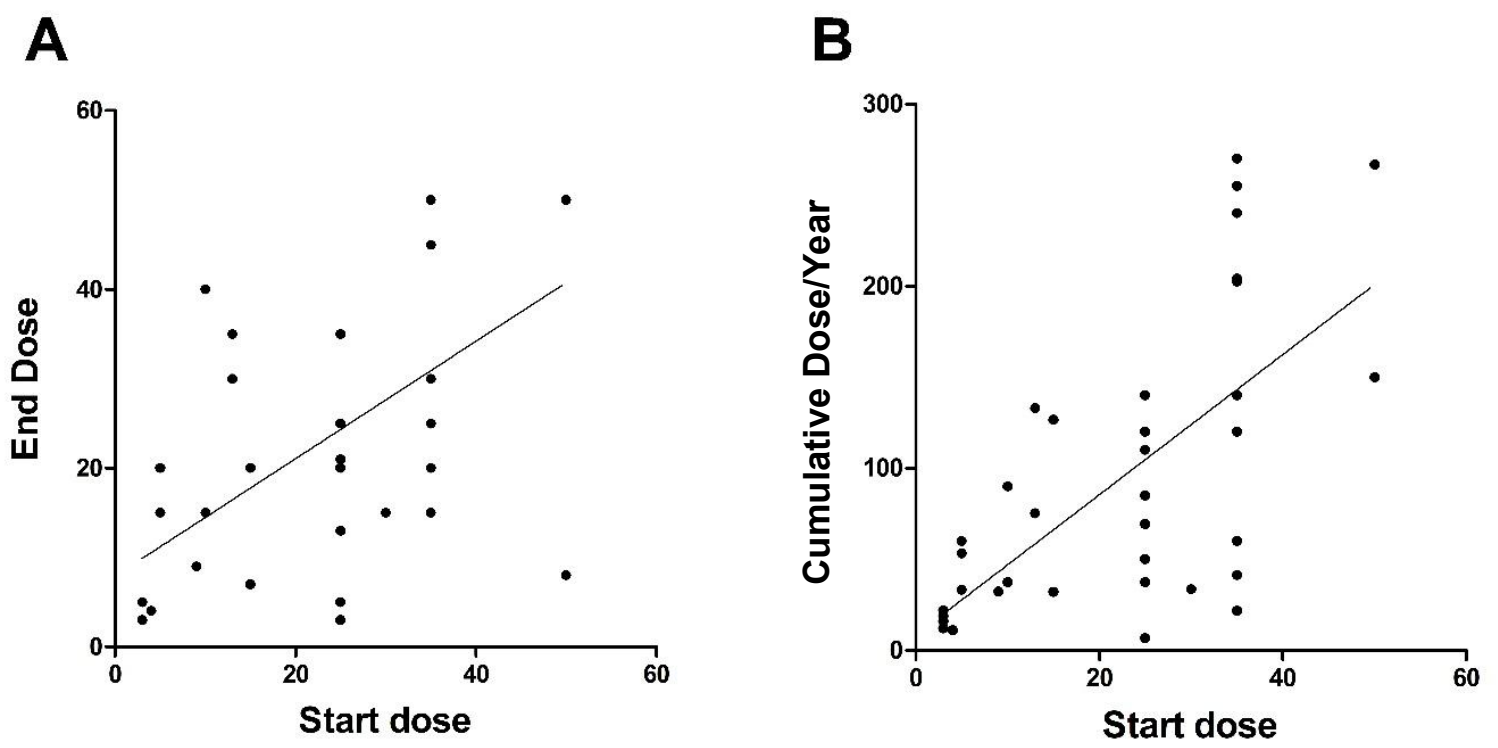

Figure 3: Relationship between the initial dose of hookworm and the last reported dose (A), and between the initial dose and the cumulative dose per year (B). For this analysis, self-reported data from individuals treating allergies and Crohn's colitis were combined and analyzed together. Correlations were statistically significant but weak for both comparisons: $\left(A, n=41, r^{2}=0.3159, p=0.0001\right)(B, n$ $\left.=41, \mathrm{r}^{2}=0.4234, \mathrm{p}=<0.0001\right)$. The average time until last dose for allergies was 1.48 years (17.72 months), and was 1.31 years (15.69 months) for Crohn's colitis. 
Self-treatment with helminths

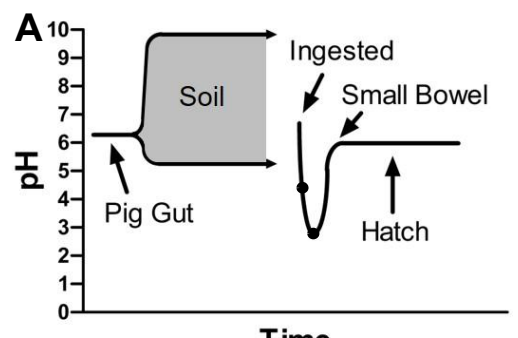

Time

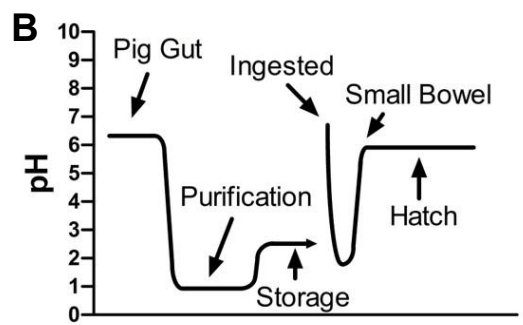

Time

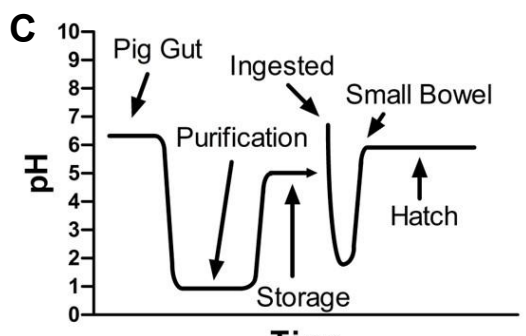

Time

Figure 4: Fluctuations in $\mathrm{pH}$ during $(\mathbf{A})$ the natural life cycle of TSO and during $(\mathbf{B}, \mathbf{C})$ two methods of preparation. After passing through the pig gut at pH 6.1-6.6, TSO is deposited in soil $[56,57]$. Geohelminths can survive in both slightly acidic and alkaline soils, with studies reporting presence of helminths between $\mathrm{pH} 5.21-9.9$ [58-60]. Ingestion in the pig occurs between approximately $\mathrm{pH} 6.7$ [61], and acidity increases as the TSO reaches the pig's anterior stomach at $\mathrm{pH} 4.4$ and then the posterior stomach at $\mathrm{pH} 2.6[57,62,63]$. As the TSO reach the pig's small bowel between pH 6.1 - 6.7, the helminth hatches. For clinical use in humans, TSO is purified from pig feces using strongly acidic conditions $(\mathrm{pH} 1)$ to inactivate viruses $(\mathrm{B}, \mathrm{C})$. After purification, the TSO is either stored at a low $\mathrm{pH}$ of $2.5(\mathrm{~B})$, or a relatively higher $\mathrm{pH}$ of 5 (C). The supplier of TSO for self-treatment asserts that only method (B) produces effective organisms, whereas the methods used in clinical trials have involved method (C) and may vary from study-to-study. TSO is ingested by humans at approximately $\mathrm{pH} 6.7[64,65]$, and reaches the stomach at pH $1.7[66,67]$. Lastly, the TSO hatches in the small bowel at pH 6 [68]. Arrows preceding breaks in the graph represent the TSO remaining in the soil or in storage for an unspecified time. 
Self-treatment with helminths

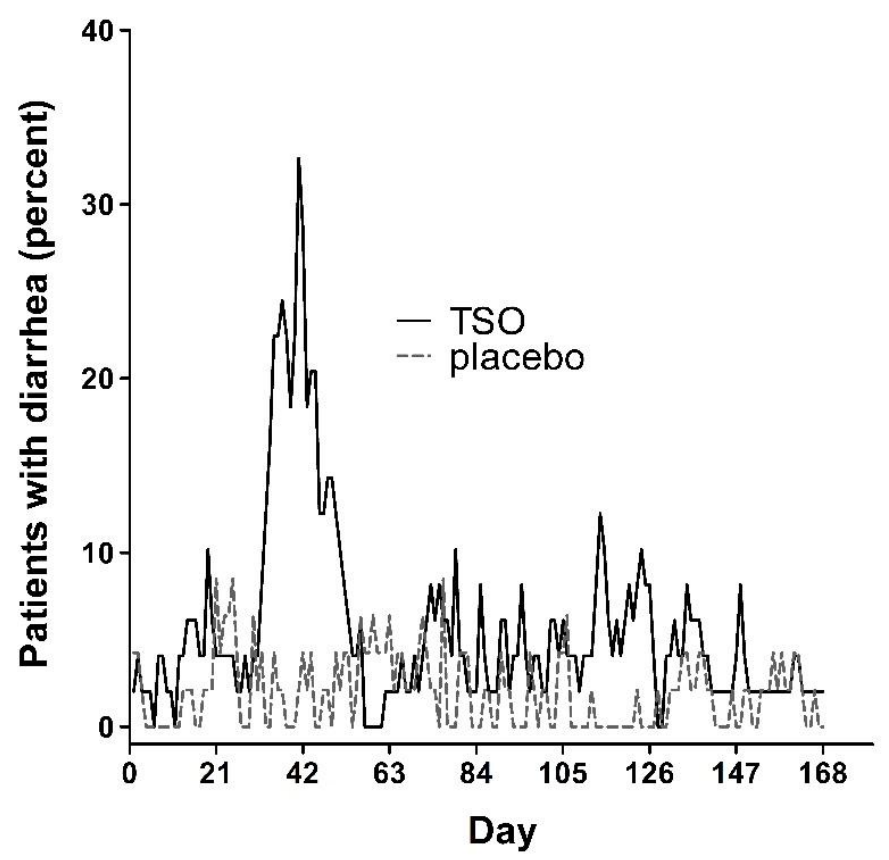

Figure 5: Percentage of patients in Bager et al.'s 2010 randomized, placebo-controlled clinical trial with TSO [43] who reported having diarrhea throughout the treatment course. For this trial, a dose of 2500 ova was taken 8 times at 21 day intervals. Days labeled in the graph indicated days in which a dose was administered, except that no dose was administered on day 168. A notable helminth-dependent increase in adverse effects peaked after the $2^{\text {nd }}$ dose, dissipating by the $4^{\text {th }}$ dose. Placebo $n=47$, $\mathrm{TSO} \mathrm{n}=49$. 
Self-treatment with helminths

\section{Tables}

\begin{tabular}{|c|c|c|}
\hline Helminth & pros & cons \\
\hline NA & $\begin{array}{l}\text {-May be more potent that other } \\
\text { helminths under some conditions, } \\
\text { especially for lung-associated } \\
\text { conditions. } \\
\text {-Least expensive of all helminths: } \\
\text { Easy to self-cultivate. } \\
\text {-Abundant data from self-treaters } \\
\text { available. }\end{array}$ & $\begin{array}{l}\text {-Beneficial effects can take months to mature: } \\
\text { tittering dose is time-prohibitive in many cases. } \\
\text {-Side effects from over-exposure can be severe. } \\
\text {-Isolated from human feces. } \\
\text {-Purification (cleaning) for FDA-approved use } \\
\text { may adversely affect the organisms' efficacy. } \\
\text {-Clinical data available, but probably do not } \\
\text { reflect optimized therapeutic approach. } \\
\text {-Utilizes blood for survival. }\end{array}$ \\
\hline TSO & $\begin{array}{l}\text { - Best tested for safety in clinical trials. } \\
\text { - Almost always non-colonizing. } \\
\text { - Minimal or no side effects when dose } \\
\text { is carefully titrated. }\end{array}$ & $\begin{array}{l}\text { Currently more expensive than other helminths. } \\
\text { Impossible to cultivate without extensive } \\
\text { resources. } \\
\text {-Can take longer than HDCs for beneficial effects } \\
\text { to mature. } \\
\text { - Clinical data available, but probably do not } \\
\text { reflect optimized therapeutic approach } \\
\text {-Isolated from pig feces. } \\
\text {-Storage conditions, especially the pH, are } \\
\text { potentially important but have not been agreed } \\
\text { upon by the scientific community. } \\
\text { - Limited data from self-treating community } \\
\text { available. }\end{array}$ \\
\hline HDC & $\begin{array}{l}\text {-Usually non-colonizing. } \\
\text {-Minimal or no side effects when dose } \\
\text { is carefully titrated. } \\
\cdot \text { Faster acting than other helminths. } \\
\text { (Beneficial effects mature faster.) } \\
\text {-Isolated from an edible host (grain } \\
\text { beetle) } \\
\text {-Abundant data from self-treaters } \\
\text { available. } \\
\text { - Does not encroach on host tissue: } \\
\text { remains in the lumen of the gut. }\end{array}$ & $\begin{array}{l}\text { - More expensive than NA. } \\
\text { - Time consuming to cultivate. Requires } \\
\text { specialized facilities. } \\
\text {-Rapid spoiling on storage, increasing cost of } \\
\text { shipping. }\end{array}$ \\
\hline TTO & -Well studied in isolated cases. & $\begin{array}{l}\text { - Can make disease worse in some cases. } \\
\text {-Time consuming and difficult to cultivate. } \\
\text {-Limited utility: Not suggested for most } \\
\text { conditions. Works well for ulcerative colitis, but } \\
\text { HDCs is also effective in those cases. }\end{array}$ \\
\hline
\end{tabular}

Table 1: pros and cons of each helminth currently used for therapeutic purposes by self-treaters. 
Self-treatment with helminths

\begin{tabular}{|l|l|l|}
\hline Helminth Supplier Name & Helminth(s) provided & Year product released \\
\hline Tanawisa & TSO & 2004 \\
\hline \multirow{3}{*}{ Autoimmune Therapies } & NA & 2007 \\
\cline { 2 - 3 } & TTO & 2007 \\
\hline \multirow{3}{*}{ Worm Therapy } & NA & 2008 \\
\cline { 2 - 3 } & TTO & 2008 \\
\cline { 2 - 3 } & HDC & 2013 \\
\hline Biome Restoration Ltd & HDC & 2013 \\
\hline \multirow{2}{*}{ Symmbio } & NA & 2016 \\
\cline { 2 - 3 } & TTO & 2016 \\
\hline \multirow{2}{*}{ The Llamas Clinic } & NA & 2018 \\
\cline { 2 - 3 } & HDC & 2018 \\
\hline \multirow{2}{*}{ YourSymbionts } & NA & 2018 \\
\cline { 2 - 3 } & TTO & 2018 \\
\hline \multirow{2}{*}{$\begin{array}{l}\text { Helminth Therapy Aust \& NZ } \\
\text { (formerly Brisbane Naturopaths) }\end{array}$} & HDC & 2019 \\
\hline Au NAturel & NA & 2019 \\
\hline
\end{tabular}

Table 2. Current helminth suppliers, helminths supplied, and the date when access began. Most of the providers listed were at one time working with other suppliers or are still affiliated with other suppliers. 
Self-treatment with helminths

\begin{tabular}{|l|c|c|c|}
\hline & \multicolumn{3}{|c|}{ Set Point (number of organisms / time) } \\
\hline & Low set point & Mid-range set point & High set point \\
\hline Hookworm (NA) & $2-5 / 3$ months & $15-20 / 3$ months & $50 / 3$ months \\
\hline Porcine whipworm (TSO) & $200 / 2$ weeks & $2500 / 2$ weeks & $7500 / 10$ days \\
\hline Rat Tapeworm (HDC) & $2-5 / 6$ weeks & $20-40 / 4$ weeks & $200 / 1$ week \\
\hline
\end{tabular}

Table 3. Dose range for helminths commonly used for therapeutic purposes. Insufficient information regarding the use of human whipworms (TTO) exists to establish set point ranges. 
Self-treatment with helminths

\section{Declarations:}

Ethical Approval and Consent to participate: Studies were approved by the Duke Institutional Review Board. Protocol number Pro00045035 and amendments.

Consent for publication: Not applicable.

Availability of supporting data: Not applicable.

Competing interests: The authors report no conflict of interest and no financial interests in helminth therapy. Authors AT, and JTS are affiliated with Duke University Medical Center, which has a vested interest in technology involving the therapeutic use of Hymenolepis diminuta.

Funding: This work was supported in part by the Immunity's Forge Foundation.

Authors' contributions: Coauthors AV, JTS and WP collected and analyzed data. WP and KJP designed the study and, with JTS and AV, drafted the manuscript. 
Self-treatment with helminths

\section{References}

1. Parker W, Perkins SE, Harker M, Muehlenbein MP. A prescription for clinical immunology: the pills are available and ready for testing. Current Medical Research and Opinion. 2012;28:1193-202. doi: 10.1185/03007995.2012.695731.

2. Parker W, Ollerton J. Evolutionary biology and anthropology suggest biome reconstitution as a necessary approach toward dealing with immune disorders. Evolution, Medicine, and Public Health. 2013;2013:89-103. doi: 10.1093/emph/eot008.

3. Villeneuve C, Kou HH, Eckermann H, Palkar A, Anderson LG, McKenney EA, et al. Evolution of the hygiene hypothesis into biota alteration theory: what are the paradigms and where are the clinical applications? Microbes Infect. 2018;20(3):147-55. Epub 2017/11/15. doi: 10.1016/j.micinf.2017.11.001. PubMed PMID: 29133248.

4. Bickler SW, DeMaio A. Western diseases: current concepts and implications for pediatric surgery research and practice. Pediatr Surg Int. 2008;24(3):251-5.

5. Preston PJ. The biology of the atopic response. Journal of the Royal Naval Medical Service. 1970;56(3):229-35. Epub 1970/01/01. PubMed PMID: 5498517.

6. Turton JA. IgE, parasites, and allergy. Lancet. 1976;2(7987):686. PubMed PMID: 60540.

7. Greenwood BM. Autoimmune disease and parasitic infections in Nigerians. Lancet.

1968;2(7564):380-2. Epub 1968/08/17. doi: 10.1016/s0140-6736(68)90595-3. PubMed PMID: 4174413.

8. Greenwood BM, Herrick EM, Voller A. Can parasitic infection suppress autoimmune disease? Proc R Soc Med. 1970;63(1):19-20. Epub 1970/01/01. PubMed PMID: 4190594; PubMed Central PMCID: PMCPMC1810949.

9. Greenwood BM, Herrick EM, Voller A. Suppression of autoimmune disease in NZB and (NZB $x$ NZW) F1 hybrid mice by infection with malaria. Nature. 1970;226(5242):266-7. Epub 1970/04/18. doi: 10.1038/226266a0. PubMed PMID: 5437516.

10. Greenwood BM, Voller A, Herrick EM. Suppression of adjuvant arthritis by infection with a strain of the rodent malaria parasite Plasmodium berghei. Ann Rheum Dis. 1970;29(3):321-3. Epub 1970/05/01. doi: 10.1136/ard.29.3.321. PubMed PMID: 5432597; PubMed Central PMCID: PMCPMC1031268.

11. Correale J, Farez M, Razzitte G. Helminth infections associated with multiple sclerosis induce regulatory B cells. Ann Neurol. 2008;64(2):187-99. PubMed PMID: 18655096.

12. Correale J, Farez M. Association between parasite infection and immune responses in multiple sclerosis. Ann Neurol. 2007;61(2):97-108. PubMed PMID: PREV200700232269.

13. Liu J, Morey RA, Wilson JK, Parker W. Practices and outcomes of self-treatment with helminths based on physicians' observations. J Helminthol. 2016;FirstView:1-11.

14. Williamson LL, McKenney EA, Holzknecht ZE, Belliveau C, Rawls JF, Poulton S, et al. Got worms? Perinatal exposure to helminths prevents persistent immune sensitization and cognitive dysfunction induced by early-life infection. Brain, Behavior, and Immunity. 2016;51:14-28. doi:

10.1016/j.bbi.2015.07.006.

15. Hewitson JP, Grainger JR, Maizels RM. Helminth immunoregulation: the role of parasite secreted proteins in modulating host immunity. Mol Biochem Parasitol. 2009;167(1):1-11.

16. Yazdanbakhsh M, Kremsner PG, van Ree R. Allergy, parasites, and the hygiene hypothesis. Science. 2002;296(5567):490-4.

17. Rook GAW. Review series on helminths, immune modulation and the hygiene hypothesis: the broader implications of the hygiene hypothesis. Immunology. 2009;126(1):3-11. PubMed PMID: 19120493.

18. Helmby H. Helminths and our immune system: Friend or foe? Parasitol Int. 2009;58(2):121-7. 
Self-treatment with helminths

19. Zaccone P, Fehervari Z, Phillips JM, Dunne DW, Cooke A. Parasitic worms and inflammatory diseases. Parasite immunology. 2006;28(10):515-23. Epub 2006/09/13. doi: 10.1111/j.13653024.2006.00879.x. PubMed PMID: 16965287; PubMed Central PMCID: PMCPmc1618732.

20. Broadhurst MJ, Leung JM, Kashyap V, McCune JM, Mahadevan U, McKerrow JH, et al. IL-22+ $\mathrm{CD} 4+\mathrm{T}$ cells are associated with therapeutic trichuris trichiura infection in an ulcerative colitis patient. Science translational medicine. 2010;2(60):60ra88. doi: 10.1126/scitranslmed.3001500. PubMed PMID: 21123809.

21. Cheng AM, Jaint D, Thomas S, Wilson J, Parker W. Overcoming evolutionary mismatch by selftreatment with helminths: current practices and experience. Journal of Evolutionary Medicine. 2015;3:Article ID 235910.

22. Smyth K, Morton C, Mathew A, Karuturi S, Haley C, Zhang M, et al. Production and Use of Hymenolepis diminuta Cysticercoids as Anti-Inflammatory Therapeutics. Journal of Clinical Medicine. 2017;6(10):98. doi: 10.3390/jcm6100098. PubMed PMID: 29064448.

23. Flowers $S$, Hopkins M. Autoimmune disease: Patients self-treat with parasitic worms. Nature. 2013;493(7431):163. doi: 10.1038/493163c. PubMed PMID: 23302848.

24. Tilp C, Kapur V, Loging W, Erb KJ. Prerequisites for the pharmaceutical industry to develop and commercialise helminths and helminth-derived product therapy. International Journal of Parasitology. 2013;43:319-25. doi: 10.1016/j.ijpara.2012.12.003.

25. Bono-Lunn D, Villeneuve C, Abdulhay NJ, Harker M, Parker W. Policy and regulations in light of the human body as a 'superorganism' containing multiple, intertwined symbiotic relationships. Clinical Research and Regulatory Affairs. 2016;33(2-4):39-48. doi: 10.1080/10601333.2016.1210159.

26. Sobotková K, Parker W, Levá J, Růžková J, Lukeš J, Jirků Pomajbíková K. Helminth Therapy - From the Parasite Perspective. Trends Parasitol. 2019;35(7):501-15. Epub 2019/06/04. doi:

10.1016/j.pt.2019.04.009. PubMed PMID: 31153721.

27. Correale J, Farez MF. The impact of parasite infections on the course of multiple sclerosis. J Neuroimmunol. 2011;233(1-2):6-11. PubMed PMID: 21277637.

28. Smallwood TB, Giacomin PR, Loukas A, Mulvenna JP, Clark RJ, Miles JJ. Helminth Immunomodulation in Autoimmune Disease. Front Immunol. 2017;8:453-. doi: 10.3389/fimmu.2017.00453. PubMed PMID: 28484453.

29. Feary JR, Venn AJ, Mortimer K, Brown AP, Hooi D, Falcone FH, et al. Experimental hookworm infection: a randomized placebo-controlled trial in asthma. Clin Exp Allergy. 2010;40(2):299-306. PubMed PMID: 20030661.

30. Kradin RL, Badizadegan K, Auluck P, Korzenik J, Lauwers GY. latrogenic Trichuris suis infection in a patient with Crohn disease. Arch Pathol Lab Med. 2006;130(5):718-20. Epub 2006/05/11. doi: 10.1043/1543-2165(2006)130[718:itsiia]2.0.co;2. PubMed PMID: 16683891.

31. Beer RJ. The relationship between Trichuris trichiura (Linnaeus 1758) of man and Trichuris suis (Schrank 1788) of the pig. Res Vet Sci. 1976;20(1):47-54. Epub 1976/01/01. PubMed PMID: 1257627.

32. Beer RJ. Experimental infection of man with pig whipworm. Br Med J. 1971;2(5752):44. Epub 1971/04/03. doi: 10.1136/bmj.2.5752.44. PubMed PMID: 5102492; PubMed Central PMCID: PMCPMC1795911.

33. Hawash MBF, Betson M, Al-Jubury A, Ketzis J, LeeWillingham A, Bertelsen MF, et al. Whipworms in humans and pigs: origins and demography. Parasites \& Vectors. 2016;9(1):37. doi: 10.1186/s13071016-1325-8.

34. Haas W, Haberl B, Syafruddin, Idris I, Kallert D, Kersten S, et al. Behavioural strategies used by the hookworms Necator americanus and Ancylostoma duodenale to find, recognize and invade the human host. Parasitol Res. 2005;95(1):30-9. Epub 2004/12/23. doi: 10.1007/s00436-004-1257-7. PubMed PMID: 15614587. 
Self-treatment with helminths

35. Looss A. Zur Lebensgeschichte des Ankylostoma duodenale. Cent Bakteriol Parasitol Infekt. $1898 ; 24: 483-8$.

36. Kou HH, Parker W. Intestinal worms eating neuropsychiatric disorders? Apparently so. Brain Res. 2018;1693(Pt B):218-21. Epub 2018/02/07. doi: 10.1016/j.brainres.2018.01.023. PubMed PMID: 29402395.

37. Summers RW, Elliott DE, Qadir K, Urban JF, Jr., Thompson R, Weinstock JV. Trichuris suis seems to be safe and possibly effective in the treatment of inflammatory bowel disease. Am J Gastroenterol. 2003;98(9):2034-41.

38. Elliott DE, Summers RW, Weinstock JV. Helminths and the modulation of mucosal inflammation. Current opinion in gastroenterology. 2005;21(1):51-8. PubMed PMID: 15687885.

39. Huang X, Zeng L-R, Chen F-S, Zhu J-P, Zhu M-H. Trichuris suis ova therapy in inflammatory bowel disease: A meta-analysis. Medicine. 2018;97(34):e12087-e. doi: 10.1097/MD.0000000000012087. PubMed PMID: 30142867.

40. Helmby $\mathrm{H}$. Human helminth therapy to treat inflammatory disorders- where do we stand? BMC Immunol. 2015;16:12. doi: 10.1186/s12865-015-0074-3. PubMed PMID: PMC4374592.

41. Wimmersberger $D$, Tritten L, Keiser J. Development of an in vitro drug sensitivity assay for Trichuris muris first-stage larvae. Parasites \& Vectors. 2013;6(1):42. doi: 10.1186/1756-3305-6-42. 42. Vejzagić $\mathrm{N}$, Adelfio R, Keiser J, Kringel H, Thamsborg SM, Kapel CMO. Bacteria-induced egg hatching differs for Trichuris muris and Trichuris suis. Parasit Vectors. 2015;8:371-. doi: 10.1186/s13071015-0986-z. PubMed PMID: 26174801.

43. Bager P, Arnved J, Ronborg S, Wohlfahrt J, Poulsen LK, Westergaard T, et al. Trichuris suis ova therapy for allergic rhinitis: a randomized, double-blind, placebo-controlled clinical trial. J Allergy Clin Immunol. 2010;125(1):123-30 e1-3. PubMed PMID: 19800680.

44. Feary J, Venn A, Brown A, Hooi D, Falcone FH, Mortimer K, et al. Safety of hookworm infection in individuals with measurable airway responsiveness: a randomized placebo-controlled feasibility study. Clinical and experimental allergy : journal of the British Society for Allergy and Clinical Immunology. 2009;39(7):1060-8. PubMed PMID: 19400893.

45. Daveson AJ, Jones DM, Gaze S, McSorley H, Clouston A, Pascoe A, et al. Effect of hookworm infection on wheat challenge in celiac disease--a randomised double-blinded placebo controlled trial. PLoS One. 2011;6(3):e17366. doi: 10.1371/journal.pone.0017366. PubMed PMID: 21408161; PubMed Central PMCID: PMC3050888.

46. Schölmerich J, Fellermann K, Seibold FW, Rogler G, Langhorst J, Howaldt S, et al. A Randomised, Double-blind, Placebo-controlled Trial of Trichuris suis ova in Active Crohn's Disease. J Crohns Colitis. 2017;11(4):390-9. doi: 10.1093/ecco-jcc/jjw184. PubMed PMID: 27707789.

47. Summers RW, Elliott DE, Urban JF, Jr., Thompson R, Weinstock JV. Trichuris suis therapy in Crohn's disease. Gut. 2005;54(1):87-90. doi: 10.1136/gut.2004.041749. PubMed PMID: 15591509; PubMed Central PMCID: PMC1774382.

48. Capron M, Béghin L, Leclercq C, Labreuche J, Dendooven A, Standaert A, et al. Safety of P28GST, a Protein Derived from a Schistosome Helminth Parasite, in Patients with Crohn's Disease: A Pilot Study (ACROHNEM). J Clin Med. 2019;9(1):41. doi: 10.3390/jcm9010041. PubMed PMID: 31878146.

49. Riveau G, Deplanque D, Remoué F, Schacht AM, Vodougnon H, Capron M, et al. Safety and immunogenicity of rSh28GST antigen in humans: phase 1 randomized clinical study of a vaccine candidate against urinary schistosomiasis. PLoS Negl Trop Dis. 2012;6(7):e1704. Epub 2012/07/18. doi: 10.1371/journal.pntd.0001704. PubMed PMID: 22802974; PubMed Central PMCID: PMCPMC3389022. 50. Riveau G, Schacht AM, Dompnier JP, Deplanque D, Seck M, Waucquier N, et al. Safety and efficacy of the rSh28GST urinary schistosomiasis vaccine: A phase 3 randomized, controlled trial in Senegalese children. PLoS NegI Trop Dis. 2018;12(12):e0006968. Epub 2018/12/12. doi: 10.1371/journal.pntd.0006968. PubMed PMID: 30532268; PubMed Central PMCID: PMCPMC6300301. 
Self-treatment with helminths

51. Sipahi AM, Baptista DM. Helminths as an alternative therapy for intestinal diseases. World J Gastroenterol. 2017;23(33):6009-15. Epub 2017/10/04. doi: 10.3748/wjg.v23.i33.6009. PubMed PMID: 28970717; PubMed Central PMCID: PMCPMC5597493.

52. Ditgen D, Anandarajah EM, Meissner KA, Brattig N, Wrenger C, Liebau E. Harnessing the Helminth Secretome for Therapeutic Immunomodulators. BioMed Research International. 2014;2014:964350. doi: 10.1155/2014/964350.

53. Motran CC, Silvane L, Chiapello LS, Theumer MG, Ambrosio LF, Volpini X, et al. Helminth Infections: Recognition and Modulation of the Immune Response by Innate Immune Cells. Front Immunol. 2018;9:664-. doi: 10.3389/fimmu.2018.00664. PubMed PMID: 29670630.

54. Sotillo J, Toledo R, Mulvenna J, Loukas A. Exploiting Helminth-Host Interactomes through Big Data. Trends Parasitol. 2017;33(11):875-88. doi: https://doi.org/10.1016/j.pt.2017.06.011.

55. Rook GAW. The hygiene hypothesis and the increasing prevalence of chronic inflammatory disorders. Trans R Soc Trop Med Hyg. 2007;101(11):1072-4.

56. Merchant HA, McConnell EL, Liu F, Ramaswamy C, Kulkarni RP, Basit AW, et al. Assessment of gastrointestinal $\mathrm{pH}$, fluid and lymphoid tissue in the guinea pig, rabbit and pig, and implications for their use in drug development. Eur J Pharm Sci. 2011;42(1-2):3-10. Epub 2010/10/12. doi:

10.1016/j.ejps.2010.09.019. PubMed PMID: 20932902.

57. Callegari M, Novais A, Oliveira E, Dias C, Schmoller D, Pereira M, et al. Microencapsulated acids associated with essential oils and acid salts for piglets in the nursery phase. Semina:Ciencias Agrarias. 2016;37:2193-208. doi: 10.5433/1679-0359.2016v37n4p2193.

58. Etewa SE, Abdel-Rahman SA, Abd El-Aal NF, Fathy GM, El-Shafey MA, Ewis AMG. Geohelminths distribution as affected by soil properties, physicochemical factors and climate in Sharkyia governorate Egypt. J Parasit Dis. 2016;40(2):496-504. Epub 2014/08/31. doi: 10.1007/s12639-014-0532-5. PubMed PMID: 27413327.

59. Sumbele IU, Ngole VM, Ekosse G-IE. Influence of physico-chemistry and mineralogy on the occurrence of geohelminths in geophagic soils from selected communities in the Eastern Cape, South Africa, and their possible implication on human health. International Journal of Environmental Health Research. 2014;24(1):18-30. doi: 10.1080/09603123.2013.782600. PubMed PMID: 93316871. Language: English. Entry Date: 20131230. Revision Date: 20190326. Publication Type: Article.

60. Amadi E, Uttah E. Impact of physico-chemical factors of contaminated foci on the survival of geohelminths in Abua Communities, Niger Delta Nigeria. Journal of Applied Sciences and Environmental Management. 2010;14(4).

61. Lewis AJ, Southern LL. Swine Nutrition: CRC Press; 2000.

62. Merchant HA, McConnell EL, Liu F, Ramaswamy C, Kulkarni RP, Basit AW, et al. Assessment of gastrointestinal $\mathrm{pH}$, fluid and lymphoid tissue in the guinea pig, rabbit and pig, and implications for their use in drug development. European Journal of Pharmaceutical Sciences. 2011;42(1-2):3-10. doi:

10.1016/j.ejps.2010.09.019.

63. Beasley DE, Koltz AM, Lambert JE, Fierer N, Dunn RR. The Evolution of Stomach Acidity and Its Relevance to the Human Microbiome. PLoS One. 2015;10(7):e0134116-e. doi: 10.1371/journal.pone.0134116. PubMed PMID: 26222383.

64. Baliga S, Muglikar S, Kale R. Salivary pH: A diagnostic biomarker. J Indian Soc Periodontol. 2013;17(4):461-5. doi: 10.4103/0972-124X.118317. PubMed PMID: 24174725.

65. Aoyama K-i, Okino Y, Yamazaki H, Kojima R, Uchibori M, Nakanishi Y, et al. Saliva pH affects the sweetness sense. Nutrition. 2017;35:51-5. doi: https://doi.org/10.1016/j.nut.2016.10.018.

66. Dressman JB, Berardi RR, Dermentzoglou LC, Russell TL, Schmaltz SP, Barnett JL, et al. Upper Gastrointestinal (GI) pH in Young, Healthy Men and Women. Pharmaceutical Research. 1990;7(7):75661. doi: 10.1023/A:1015827908309. 
Self-treatment with helminths

67. Foltz E, Azad S, Everett ML, Holzknecht ZE, Sanders NL, Thompson JW, et al. An assessment of human gastric fluid composition as a function of PPI usage. Physiol Rep. 2015;3(1). Epub 2015/01/30. doi: 10.14814/phy2.12269. PubMed PMID: 25626870; PubMed Central PMCID: PMCPMC4387745.

68. Fallingborg J. Intraluminal pH of the human gastrointestinal tract. Dan Med Bull. 1999;46(3):18396. Epub 1999/07/28. PubMed PMID: 10421978. 